\title{
PREVALENCIA DE CONDUCTAS ALIMENTARIAS DE RIESGO EN ADOLESCENTES DE CARACAS: 2012 VS. 2018.
}

\author{
PREVALENCE OF RISK EATING BEHAVIOR IN YOUTH FROM CARACAS: \\ 2012 VS. 2018
}

Zerpa García Carlos Enrique ${ }^{1}$, Ramírez Andreína².

1 Universidad Simón Bolívar, Venezuela. 2 Fundación Ayúdate, Caracas, Venezuela.

Citation: Zerpa García C.E., Ramírez A., (2020) Prevalencia de Conductas Alimentarias de Riesgo en Adolescentes de Caracas: 2012 vs. 2018. Revista Salud Pública y Nutrición, 19 (2), 9-18.

Editor: Esteban G. Ramos Peña, Dr. CS., Universidad Autónoma de Nuevo León, Facultad de Salud Pública y Nutrición, Monterrey Nuevo León, México. Copyright: (C2020 Zerpa García C.E., et al. This is an open-access article distributed under the terms of Creative Commons Attribution License [CC BY 4.0], which permits unrestricted use, distribution, and reproduction in any medium, provided the original author and source are credited.

Competing interests: The authors have declared that no competing interests exist.

DOI: https://doi.org/10.29105/respyn19.2-2

Recibido: 24 de marzo 2020; $\quad$ Aceptado: 15 de junio 2020

Email: czerpa@usb.ve 


\title{
PREVALENCIA DE CONDUCTAS ALIMENTARIAS DE RIESGO EN ADOLESCENTES DE CARACAS: 2012 VS. 2018.
}

\author{
Zerpa García Carlos Enrique ${ }^{1}$, Ramírez Andreína². \\ 11 Universidad Simón Bolívar, Venezuela. 2 Fundación Ayúdate, Caracas, Venezuela.
}

\begin{abstract}
RESUMEN
Introducción: Los trastornos de la conducta alimentaria (TCA) constituyen un grupo de desórdenes psicológicos graves. Dentro del mismo, anorexia y bulimia han sido clasificados en el DSM V (APA, 2013) como trastornos específicos. Objetivo: Comparar la prevalencia de las conductas alimentarias de riesgo sugestivas de trastornos del comportamiento alimentario en adolescentes de un municipio de la ciudad de Caracas en dos muestras tomadas en años diferentes (NA: 2012 vs NB: 2018). Material y Método: Los participantes provenían de seis (6) institutos educativos de Caracas; fueron evaluados con el cuestionario EAT-26 (Ntotal = 933; NA = 311; 40,51\% hombres; 59,49\% mujeres); (NB = 622; 40,51\% hombres; 59,49\% mujeres), con edad promedio $N A=16,88$ años y $N B=14,88$ años. Se hicieron comparaciones no paramétricas empleando la prueba U de Mann-Whittney. Resultados: Se observó prevalencia de TCA (para EAT-26 $\geq 20$ ) de 6,4\%, (año 2012) y 15,3\% (año 2018), así como diferencias estadísticamente significativas en los puntajes totales del EAT-26 para los factores Bulimia y Control Oral, pero no para Dieta; y para puntaje total y Control Oral; solo se observaron diferencias significativas en dos (2) de los seis (6) institutos de educación considerados. Conclusiones: Los resultados apuntan hacia la importancia de considerar estos cambios reportados en torno a la prevalencia de conductas de riesgo de TCA.

Palabras Clave: Trastornos del comportamiento alimentario; estudiantes de bachillerato; escala EAT-26; pruebas no-
\end{abstract} paramétricas.

\section{ABSTRACT}

Introduction: Eating disorders (ATCS) are a group of serious psychological disorders. Within it, anorexia and bulimia have been classified in DSM V (APA, 2013) as specific disorders. Objective: To compare the prevalence of risky eating behaviors suggestive of Eating Disorders in adolescents from a municipality in the city of Caracas in two samples taken in different years (NA: 2012 vs NB: 2018). Material and method: The participants came from six (6) educational institutes of Caracas; they were evaluated with the EAT-26 questionnaire (Ntotal = 933; NA = 311; 40.51\% men; 59.49\% women); (NB = 622; 40.51\% men; $59.49 \%$ women), average age $N A=16.88$ years and $N B=14.88$ years. Nonparametric comparisons were made using the MannWhittney $U$ test. Results: A prevalence of eating disorders was observed (for EAT-26 $\geq 20$ ) of 6.4\%, (year 2012) and 15.3\% (year 2018), as well as statistically significant differences in the total scores of the EAT-26 for the Bulimia and Oral Control factors, but not for Diet; and for total score and Oral Control; significant differences were only observed in two (2) of the six (6) educational institutes considered. Conclusions: The results point to the importance of considering these reported changes around the prevalence of TCA risk behaviors.

Key words: Risk eating behaviors; high school students; EAT-26 scale; non-parametric tests. 


\section{Introducción}

Los trastornos de la conducta alimentaria (TCA) constituyen un grupo de desórdenes psicológicos graves. Dentro del mismo, anorexia y bulimia han sido clasificados en el DSM V (APA, 2013) como trastornos específicos, incorporando también al Trastorno por atracón, y en ellos la calificación de gravedad mínima se fundamenta en un elemento clave de diagnóstico: el índice de masa corporal (IMC) en el caso de la anorexia nerviosa; la frecuencia de comportamiento compensatorio inapropiado en la bulimia; y la frecuencia de excesos de ingesta de comida en el Trastorno por atracón (Walsh, 2019). Estudios recientes confirman que la prevalencia mundial es alta, especialmente en mujeres y su tasa aumentó desde 3,5\% para el período $2000-2006$, hasta $7,8 \%$ para el período 2013-2018 (Galmiche, Déchelotte, Lambert \& Tavolacci, 2019). Esto representa un verdadero desafío, tanto para las políticas públicas de salud como para los proveedores de servicios de salud, puesto que resultan potencialmente peligrosos al tener consecuencias físicas y emocionales y su pronóstico de cura es reservado si no se atienden tempranamente.

Estos trastornos tienen serios impactos tanto económicos como sociales y, de acuerdo con Vaidyanathan, Patnaik \& Menon (2019), pueden ser comparables con los impactos que tienen trastornos como la ansiedad y la depresión; señalan además que desde fines del siglo XVII ya se empleaba el término "consunción nerviosa", para referirse a ellos, siendo percibidos históricamente como síndromes vinculados a la cultura occidental y fueron descritos por primera vez en mujeres caucásicas de América del Norte y el oeste de Europa (Pike, Hoek \& Dunne, 2014); actualmente se han identificado en todas las culturas del mundo con tasas de prevalencia más bajas que en los países occidentales, aunque en franco crecimiento (Hoek, 2014).

En efecto, en años recientes han sido numerosos los estudios de TCA que se han efectuado en diversos lugares. La literatura reporta un interés destacado en el tema, hecho comprobable en investigaciones como las de Dao-Le, Barendregt, Hay \& Mihalopoulos (2017) en la que reportan un meta-análisis de 112 investigaciones empíricas publicadas entre los años 2009 a 2015 y solo en el tema de la prevención. Muchos estudios se han enfocado en la prevalencia de estos trastornos, por ejemplo, el de Cheah, Jackson, Touyz \& Hay (2020, en prensa) en Oceanía; Keski-Rahkonen \& Mustelin (2016) en Europa; van Hoeken, Burns \& Hoek (2016) en África; Thomas, Lee \& Becker (2016) en Asia; y Kolar, Rodríguez, Chams \& Hoek (2016) en América Latina.

En esta última región, son numerosas las investigaciones en las que la población objetivo han sido los adolescentes (p. e.: Caldera, del Campo, Caldera, Reynoso \& Zamora, 2019, en México; Montoya \& Angamarca, 2016, en Ecuador; MorenoGutiérrez et al, 2016, en Colombia; Urrutia, Cazenave, Badillo \& Fajardo, 2008, en Chile; entre otros). Las tasas de prevalencia para América Latina son comparables entre países como Colombia, Brasil, Venezuela y México, observándose tasas promedio de $1,16 \%$ para anorexia y bulimia en la región (Kolar et al, 2016). Hoek (2014) refiere que la evidencia actual sugiere que las tasas para bulimia nerviosa están disminuyendo en los países occidentales y se mantiene estable para la anorexia nerviosa. En el caso particular de Venezuela, se han reportado muy pocos estudios de TCA, muy disímiles, encontrándose prevalencias de entre 01,56\% (Quintero-Párraga, Pérez-Montiel, MontielNava, Pirela, Acosta \& Pineda, 2003), hasta 18\% (Ríos, Machado, Rísquez, \& Ortega, 1994), a través de medidas realizadas con el instrumento EAT-26, y no existen reportes que comparen muestras no relacionadas de adolescentes que hayan sido evaluados en momentos distintos pero en las mismas instituciones educativas para obtener la prevalencia de conductas sugestivas de estos trastornos y su cambio en el tiempo. Resulta importante dar cuenta de ello puesto que los aspectos epidemiológicos de los TCA resultan en información útil para aspectos de identificación de prioridades en su representación conveniente para procesos de adjudicación de recursos financieros dirigidos a las propuestas de intervención y en especial, prevención de tales trastornos. De allí que el objetivo de esta investigación fue comparar en dos años diferentes (2012 vs 2018) las tasas de prevalencia de conductas sugestivas de riesgo de TCA (EAT-26 $\geq 20$ ), con miras a las implicaciones que la prevalencia tiene para las políticas de atención de estos trastornos en adolescentes en un municipio de la ciudad de Caracas, Venezuela. 


\section{Material y Método}

La población se constituyó por las personas de edad adolescente que cursaban estudios de educación secundaria en el municipio Sucre, autarquía perteneciente al Distrito Metropolitano de Caracas, ( $\mathrm{N}=457935,50,93 \%$ y $49,07 \%$ mujeres; INE, 2014). Las muestras fueron de tipo probabilísticas; para ello se seleccionó dentro del municipio la parroquia con mayor índice poblacional, correspondiéndose con la parroquia Leoncio Martínez $(\mathrm{N}=63118)$ y en ella, los institutos educativos con mayor tasa de estudiantes; esto incluyó a seis (6) instituciones educativas, en las que se hizo selección aleatoria de los integrantes de cada muestra, lo cual se aprecia en la tabla 1. Los criterios de inclusión se correspondieron con: a) ser estudiante del tercer nivel de educación básica o del nivel de educación media diversificada; b) pertenecer a la comunidad educativa de los institutos seleccionados; el criterio de exclusión fue ser menor de 12 años o mayor de 18. El tamaño muestral se estimó a partir de la ecuación:

$$
n=\frac{4 * N * N^{*} q}{E^{2}(N-1)+4 * p * q}
$$

n: es el tamaño muestral a estimar; 4: es una constante; $p$ y q: son las probabilidades de éxito o fracaso que tienen un valor de $50 \%$, por lo que p y q=50; N: tamaño de la población; es el error muestral seleccionado (con el valor de 5\%).

La muestra para el año 2012 quedó conformada por $\mathrm{NA}=311$ participantes y la muestra del año 2018 por $\mathrm{NB}=622$. Esta última tomó en cuenta los mismos institutos encuestados en el año 2012 a fin de controlar el tipo de institución y hacer comparables las medidas en el tiempo; resultó mayor debido a un incremento de la matrícula en las instituciones públicas participantes, proveniente de la movilidad de estudiantes desde otras instituciones privadas. $\mathrm{La}$ investigación fue de tipo descriptivo, no experimental y de naturaleza ex post facto retrospectivo, en tanto buscaba especificar propiedades y características importantes del fenómeno objeto de análisis, describiendo las tendencias en las variables de los grupos que se estudiaron, (Hernández, Fernández \& Baptista, 2014). Los estudiantes participaron de forma voluntaria y completaron en todas sus partes tanto una hoja de datos socio-demográficos como el instrumento EAT-26.
Instrumento

Cuestionario de Actitudes Alimentarias EAT-26. Se administró el EAT-26; se trata de un instrumento de auto-informe, versión abreviada de 26 ítems del EAT-40, elaborado originalmente por Garner, Olmsted, Bohr \& Garfinkel (1982) que mide la presencia de conductas de riesgo de Trastornos del comportamiento alimentario; adaptado en Venezuela, por Ríos, Machado, Rísquez \& Ortega (1994) en una muestra de 330 jóvenes de ambos sexos, reportó un coeficiente alpha de Cronbach $\alpha=$ 0,76 y estimación de confiabilidad test-retest $\mathrm{de} \mathrm{rtt}=$ 0,72 para la escala total. Se presentan los planteamientos en una escala de tipo Likert, de elección forzada de 6 puntos que van desde 1 ("nunca") hasta 6 ("siempre). La puntuación total se obtiene recodificando puntajes de la siguiente manera: puntajes de 1 a 3 son recodificados como 0 ; 4 se recodifica como 1; 5 como 2 ; y 6 se recodifica como 3, con la excepción de los ítems 19 y 25, los cuales son calificados de manera inversa.

La puntuación total de EAT-26 oscila entre 0 y 78 y los autores del instrumento han establecido un corte en 20 puntos que se utiliza para determinar casos de conductas alimentarias de riesgo de las de no-riesgo. Este mismo punto de corte fue reportado en el estudio de Ríos et al. El instrumento contiene a su vez tres componentes o factores; conforman las sub-escalas de Dieta (13 ítems referidos a comportamientos de evitación de comestibles que incrementen el peso corporal y preocupación por la consunción o bien, la distorsión de la imagen corporal); Bulimia (seis ítems sobre tendencia al comportamiento voraz en la alimentación y conductas bulímicas); y Control Oral (siete ítems referidos al auto control de la ingesta de alimentos y la presión proveniente del entorno social).

Las propiedades psicométricas que se han reportado para el instrumento original en idioma Inglés refieren estabilidad en la medida en el tiempo (test-retest a dos y cuatro años) que oscila entre $\mathrm{rtt}=0,84 \mathrm{y} \mathrm{rtt}=$ 0,89 (Banasiak, Wertheim, Koerner \& Voudouris, 2001; Carter \& Moss, 1984); un coeficiente alpha de Cronbach $\quad \alpha=0,938$ para la escala total con muestras de mujeres españolas (Ribas, Bersabé, Jiménez \& Berrocal, 2010); y $\alpha=0,921, \alpha=0,891, \alpha$ $=0,857$ y $\alpha=0,818$ para la escala total, factor 
Bulimia, factor Dieta y factor Control, respectivamente, en muestras colombianas (Constaín, et. al, 2014).

Análisis de los datos

Para analizar los datos se empleó el programa SPSS v.23 y se estimaron estadísticos descriptivos de tendencia central en las variables de naturaleza numérica; debido a la ausencia de normalidad en las distribuciones de puntajes de la medida del EAT-26 y la existencia de valores atípicos, se empleó la prueba no paramétrica U de Mann-Whitney de muestras independientes para el cálculo de las diferencias de medianas del instrumento entre los grupos de 2012 y 2018, y también por institución educativa.

Procedimiento

El estudio implicó las siguientes etapas: 1) gestión de la autorización para la administración de los instrumentos, ante autoridades y padres, en los institutos educativos en los que se efectuó la investigación; 2) solicitud de participación voluntaria de los estudiantes; 3 ) administración de la hoja de datos socio-demográficos y del instrumento EAT-26; 4) elaboración de una base de datos para SPSS v.23; y 5) Análisis de los datos recopilados. Para salvaguardar los necesarios atributos éticos que están implicados en la investigación, a cada participante se le solicitó su autorización para que se le administrara el instrumento y suministrara información socio-demográfica para los propósitos que se perseguían. El tratamiento que se le dio a la información recabada fue de estricta confidencialidad. En ningún momento se dieron a conocer en el informe de la investigación los nombres de las personas participantes; solo se indicaron los nombres de las instituciones educativas específicas a las que pertenecían los estudiantes encuestados. La investigación consideró los lineamientos éticos para el trabajo con participantes humanos expuestos por Kerlinger y Lee (2002; p. 396 y siguientes) en tanto no se puso en riesgo a las personas ni se violaron las normas del libre consentimiento informado, salvaguardándose el bienestar del grupo de participantes al cuidar que no existieran riesgos explícitos ni potenciales para las personas.

\section{Resultados}

Del total de $\mathrm{N}=933$ adolescentes (tabla 1), el grupo que conformó la muestra de la recolección de datos del año 2012 presentó los siguientes atributos: NA= 311 participantes $(33,33 \%)$ con edades comprendidas entre 15 y 18 años $(40,51 \%$ hombres con $\mathrm{Mm}=16,44$ y DTm $=0,754 ; 59,49 \%$ mujeres con $\mathrm{Mf}=16,67$ y $\quad \mathrm{DTf}=0,776$ ); de otro lado, el grupo que conformó la muestra del año 2018 se caracterizó por lo siguiente: $\mathrm{NB}=622(66,66 \%)$, con edades comprendidas entre 12 y 18 años $(40,51 \%$ hombres con $\mathrm{Mm}=15,04$ y DTm= 1,587; 59,49\% mujeres con $\mathrm{Mf}=14,77$ y $\mathrm{DTf}=1,592)$.

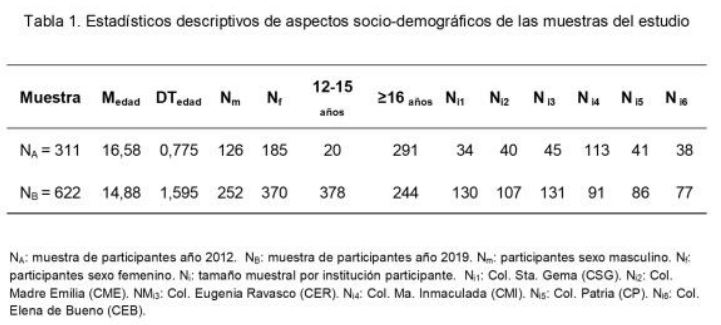

La figura 1 presenta los gráficos elaborados para la evaluación de los atributos de las distribuciones de puntajes en las muestras seleccionadas, para la escala total del EAT-26. Puede apreciarse en dicho gráfico que las formas de las distribuciones de los datos difieren de la curva normal, mostrándose coleadas hacia la derecha, con asimetría positiva (NA: As = $1,487 ; \mathrm{e}=0,138$, y NB: As $=1,781 ; \mathrm{e}=0,098)$ y de forma leptocúrtica (NA: $\mathrm{K}=3,220$; e $=0,276$, y NB: $\mathrm{K}=4,577 ; \mathrm{e}=0,196)$. Igualmente, la prueba de Levene sugiere no equivalencia entre las varianzas de los puntajes del EAT-26 para las muestras NA y NB (para EAT escala total $\mathrm{W}=31,009, \mathrm{p}<0,05$; Dieta: $\mathrm{W}$ $=4,453, \mathrm{p}<0,05 ;$ Bulimia: $\mathrm{W}=38,719 \mathrm{p}<0,05$; Control Oral $W=3,885 \mathrm{p}<0,05)$. Lo anterior sugirió la necesidad de realizar análisis no paramétricos a los datos obtenidos con la administración del instrumento EAT-26. Dado que un importante número de observaciones en las muestras NA y NB obtuvo puntajes superiores al percentil P75, las distribuciones se encuentran coleadas a la derecha del gráfico. Tanto las figuras 1 y 2 como la tabla 2 muestran alta variabilidad en los puntajes del grupo evaluado en el año 2012 y del grupo del año 2018. 
Trastornos del comportamiento alimentario; estudiantes de bachillerato; escala EAT-26; pruebas no-

paramétricas.

Artículo Original

Figura 1. Gráficos de normalidad de las distribuciones de puntajes en EAT-26 (total) para las muestras del estudio.
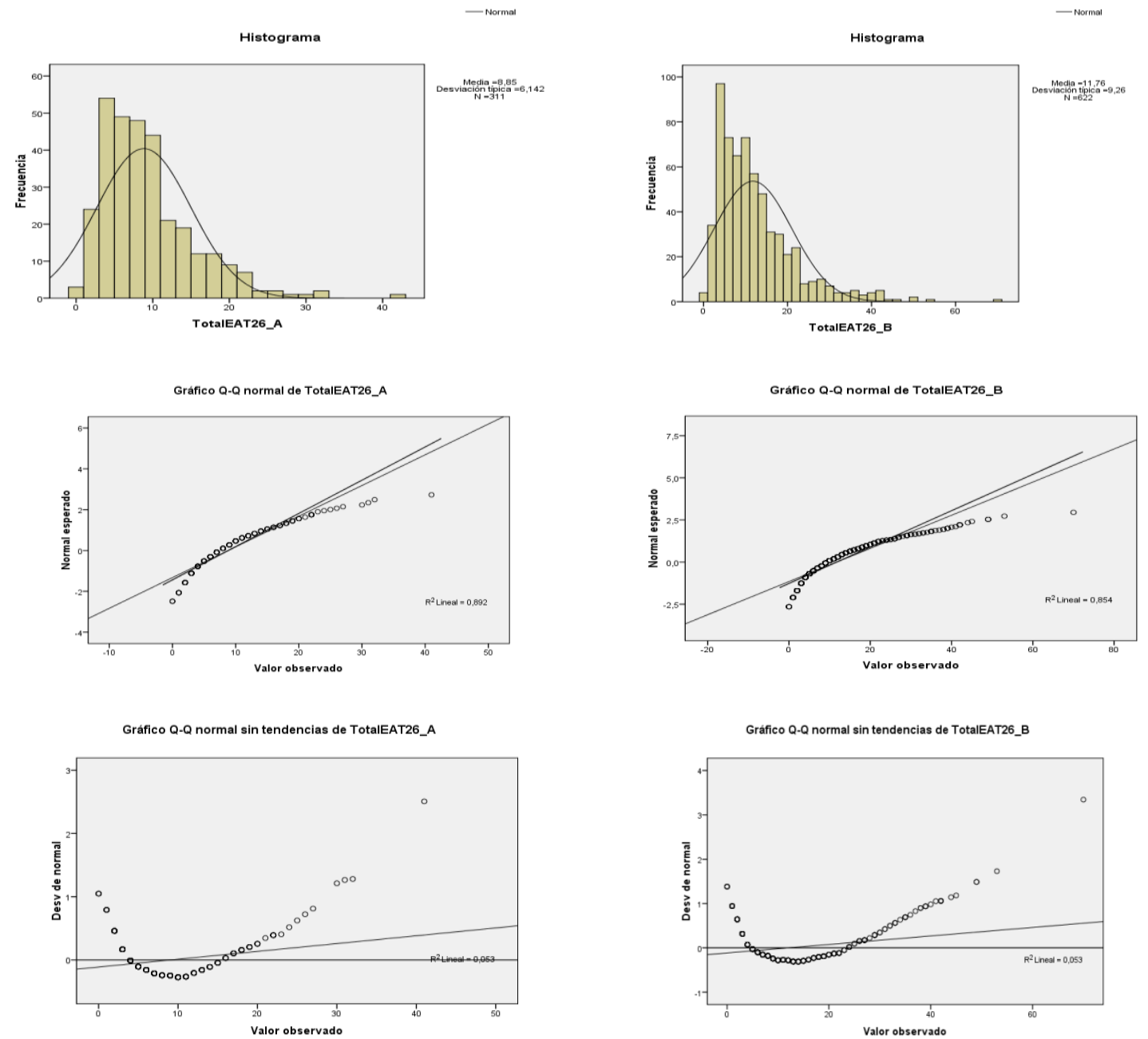

Figura 2. Gráficos de caja y bigotes de las distribuciones de puntajes en EAT-26 (total) para las muestras del estudio.
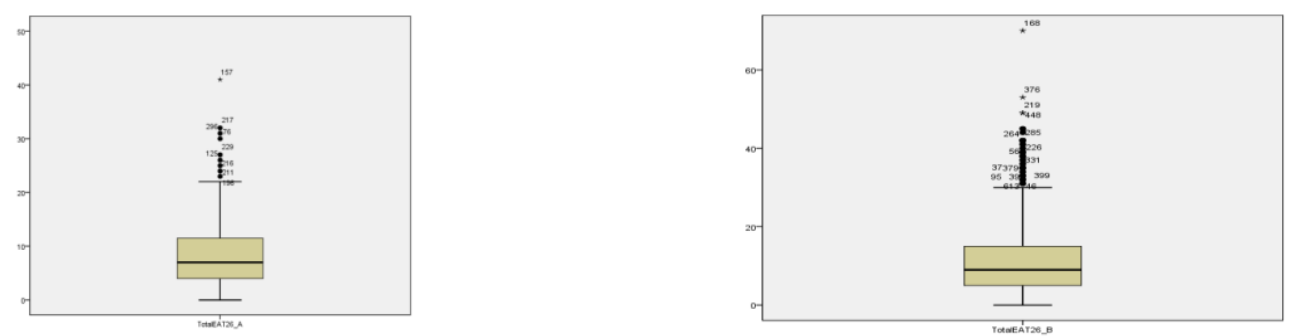
Tabla 2. Estadisticos descriptivos para el EAT-26, escala total

\begin{tabular}{|c|c|c|c|c|c|c|c|c|}
\hline Muestra & $M_{\mathrm{tot}}$ & $D_{\text {tot }}$ & $M_{\text {Dieta }}$ & $\mathrm{DT}_{\text {Dieta }}$ & M Bulimia & $\mathrm{DT}_{\text {Buimia }}$ & $\mathrm{M}_{\text {control Oral }}$ & $D T_{\text {Contiol Oral }}$ \\
\hline$N_{A}=311$ & 8,85 & 6,142 & 5,02 & 4,811 & 0,74 & 1,545 & 3,09 & 2,691 \\
\hline$N_{B}=622$ & 11,76 & 9,260 & 5,09 & 5,482 & 1,45 & 2,665 & 4,18 & 2,872 \\
\hline
\end{tabular}

$\mathrm{N}_{\mathrm{A}}$ : muestra de participantes año 2012. $\mathrm{N}_{\mathrm{B}}$ : muestra de participantes año 2019

Alta variabilidad de los datos, tanto para la escala total como para los factores componentes, también se observa en la tabla 3 , en la cual se presentan los descriptivos obtenidos solo para el subgrupo de participantes que obtuvo en el EAT-26, puntajes $\geq$ 20 , conformando el grupo con riesgo de trastornos del comportamiento alimentario (TCA) conforme a los presupuestos del modelo de medida. En dicha tabla se muestra que las desviaciones típicas tienden a ser más amplias para el grupo del año $2018\left(\mathrm{~N}_{\mathrm{B}}\right)$, por lo que se trata de una muestra menos homogénea que la del año $2012\left(\mathrm{~N}_{\mathrm{A}}\right)$.

Tabla 3. Estadisticos descriptivos para el EAT-26, puntajes $\geq 20$ (riesgo de TCA)

\begin{tabular}{lllllllll}
\hline Muestra & $M_{\text {total }}$ & $D_{T_{\text {total }}}$ & $M_{\text {Dieta }}$ & $D_{\text {Dieta }}$ & $M_{\text {Bulimia }}$ & $D_{\text {Bulmial }}$ & $M_{\text {Control Oral }}$ & $D_{\text {Control Oral }}$ \\
\hline$N_{A}=20$ & 24,60 & 5,345 & 16,10 & 5,955 & 3,80 & 3,27 & 4,70 & 4,028 \\
$N_{B}=95$ & 28,97 & 8,980 & 14,54 & 6,234 & 4,56 & 4,61 & 7,73 & 3,645 \\
\hline
\end{tabular}

Al contrastar las medianas de las distribuciones de puntajes, en la tabla 4 a se aprecia el comportamiento de los datos para los puntajes de la escala total y sus factores; y en la tabla $4 \mathrm{~b}$ igualmente se aprecia el comportamiento de los datos para el subgrupo de riesgo de TCA (EAT-26, puntajes $\geq 20$ ). Con un nivel de confianza del $95 \%$, se observan diferencias estadísticamente significativas entre la recolección de datos de los años 2012 y 2018 en el caso de los puntajes de escala total $(\mathrm{U}=80077,5$; sig. $=0,000$; $\mathrm{p}<0,05)$, y en los factores de Bulimia $(\mathrm{U}=82545,5$; sig. $=0,000 ; \mathrm{p}<0,05)$ y Control Oral $(\mathrm{U}=72323,5$; sig. $=0,000 ; \mathrm{p}<0,05)$; particularmente en el caso de los puntajes del grupo de riesgo de TCA se observan diferencias estadísticamente significativas para las muestras NA y NB en el puntaje total del instrumento EAT-26 $(\mathrm{U}=666,00 ;$ sig. $=0,035 ; \mathrm{p}<0,05)$ y en el factor Control Oral $(\mathrm{U}=525,5 ;$ sig. $=0,002$; $\mathrm{p}<0,05)$.
Tabla 4a. Contrastes de medianas para los niveles de las variables del estudio (U de Mann-Whitney)

\begin{tabular}{|c|c|c|c|c|c|c|c|c|}
\hline & \multicolumn{2}{|c|}{ Total_EAT26 } & \multicolumn{2}{|c|}{ Dieta_EAT26 } & \multicolumn{2}{|c|}{ Bulimia_EAT26 } & \multicolumn{2}{|c|}{ ControlOral_EAT26 } \\
\hline Grupo & $\mathrm{RM}$ & U & RM & U & $\mathrm{RM}$ & U & $\mathrm{RM}$ & U \\
\hline $\begin{array}{l}\text { NA } \\
\text { NB }\end{array}$ & $\begin{array}{l}413.48 \\
493.76\end{array}$ & $80077.5^{\star}$ & $\begin{array}{l}479.2 \\
460.9\end{array}$ & 92927 & $\begin{array}{l}421.4 \\
489.8\end{array}$ & $82545.5^{*}$ & $\begin{array}{l}388.6 \\
506.2\end{array}$ & $72323.5^{\star}$ \\
\hline
\end{tabular}

RM: Rango Promedio; $"=p<0,05$. $\mathrm{N}_{\mathrm{A}}$ : muestra de participantes año 2012. $\mathrm{N}_{\mathrm{B}}$ : muestra de participantes año 2019. ${ }^{1}$ Medida del EAT-26220.

Tabla 4b. Contrastes de medianas para los niveles de las variables del estudio para EAT$26 \geq 20$ (U de Mann-Whitney)

\begin{tabular}{|c|c|c|c|c|c|c|c|c|}
\hline \multirow{2}{*}{ Grupo } & \multicolumn{2}{|c|}{ EAT-26 $220^{1}$} & \multicolumn{2}{|c|}{ EAT26_Dieta $20{ }^{1}$} & \multicolumn{2}{|c|}{ EAT26_Bulimia $22{ }^{1}$} & \multicolumn{2}{|c|}{ EAT26_Control Oral 220} \\
\hline & RM & U & RM & U & RM & U & RM & U \\
\hline NA & 43,80 & \multirow{2}{*}{$666.00^{*}$} & 65,10 & \multirow{2}{*}{808,00} & 53,10 & \multirow{2}{*}{852,00} & 36,78 & \multirow{2}{*}{$525.5^{\star}$} \\
\hline NB & 60,99 & & 56,51 & & 59,03 & & 62,47 & \\
\hline
\end{tabular}

La tabla 5 presenta los contrastes de las medianas de los puntajes del EAT-26, escala total, para las muestras NA y NB, y de acuerdo al instituto educativo de procedencia. En la misma puede apreciarse que en cinco (5) de los seis (6) institutos se presentan diferencias estadísticamente significativas para este contraste. En efecto, se observan diferencias para el instituto CSG en el factor Dieta ( $U=1537,5$; sig. $=0,006 ; \mathrm{p}<0,05)$; instituto CME tanto para el puntaje total de la prueba $(\mathrm{U}=1443,5 ;$ sig. $=0,002 ; \mathrm{p}<0,05)$, como en los factores de Bulimia $(\mathrm{U}=1587$; sig. $=0,006 ; \mathrm{p}<0,05)$ y Control Oral $(\mathrm{U}=1462 ;$ sig. $=0,003 ; \mathrm{p}<0,05)$; instituto CER, tanto en el factor Bulimia $(\mathrm{U}=2106$; sig. $=0,019 ; \mathrm{p}<0,05)$ como en Control Oral $(\mathrm{U}=$ 1824; sig. $=0,001 ; \mathrm{p}<0,05)$; instituto $\mathrm{CP}$ para el puntaje total $\quad(U=1192 ;$ sig. $=0,003 ; \mathrm{p}<0,05)$, para el factor Bulimia $(\mathrm{U}=1404$; sig. $=0,027$; $\mathrm{p}<0,05)$ y el factor Control Oral $(\mathrm{U}=1287$; sig. $=$ 0,013; $<<0,05)$; $y$, finalmente, para el instituto CEB solo en el factor Bulimia $(\mathrm{U}=1053$; sig. $=0,006$; $\mathrm{p}<0,05)$.

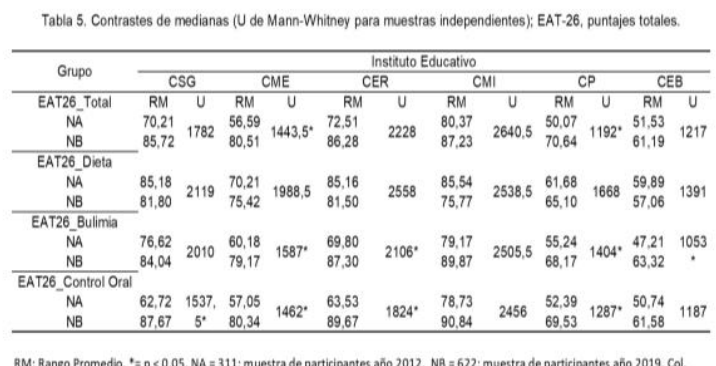


En la tabla 6 se aprecian los contrastes efectuados a las medianas de las muestras por instituto, en el subgrupo de riesgo de TCA (EAT-26 $\geq 20$ ). Se evidencia que para este subgrupo existen diferencias estadísticamente significativas para las medidas del EAT-26 del factor Control Oral en el instituto CME $(\mathrm{U}=6,00 ;$ sig. $=0,035 ; \mathrm{p}<0,05)$ y para el factor Dieta en el instituto CMI $(U=17,5$; sig. $=0,022 ; \mathrm{p}<0,05)$, no observándose diferencias en ningún otro factor entre instituciones educativas.

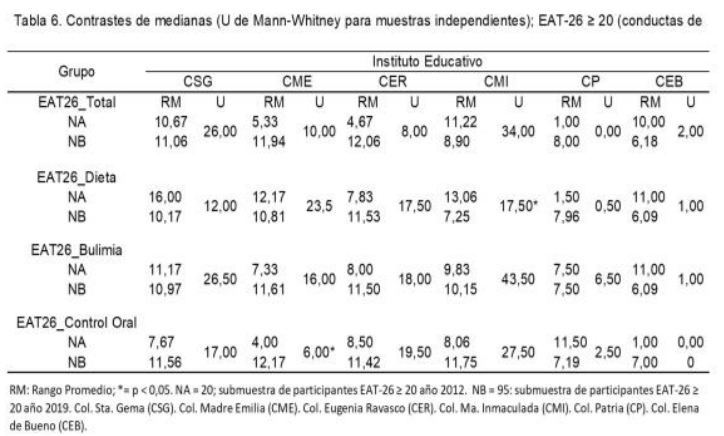

Finalmente se estimó la prevalencia de conductas de riesgo sugestivas de TCA a través del EAT-26 (EAT$26 \geq 20)$ obteniéndose una tasa de $6,43 \%$, (NA $=20)$ para la muestra del año 2012; 16 casos se corresponden con estudiantes femeninas $(5,14 \%)$ y 4 con estudiantes masculinos $(1,29 \%)$; una tasa de $15,3 \%(\mathrm{NB}=95)$ para la muestra del año 2018), 70 casos correspondientes a estudiantes femeninas $(11,25 \%)$ y 25 casos a estudiantes masculinos $(4,02 \%)$. Las medianas de estas dos distribuciones son estadísticamente distintas al estimar la prueba de Brown-Forsythe en la que tal diferencia se observa para la distribución de EAT-26 del grupo total $(\mathrm{F}=$ $32,638 \mathrm{p}<0,05)$ y para las distribuciones de Bulimia $(\mathrm{F}=26,923 ; \mathrm{p}<0,05)$ y Control Oral $(\mathrm{F}=32,520$; $\mathrm{p}<0,05)$ del grupo total, excepto para Dieta $(\mathrm{F}=$ $0,035 ; \mathrm{p}>0,05)$.

\section{Discusión}

La investigación realizada comparó a través del instrumento EAT-26, las conductas de riesgo asociadas a los trastornos del comportamiento alimentario en estudiantes adolescentes de ambos sexos de un municipio de la ciudad de Caracas, Venezuela, con datos recolectados en dos (2) momentos diferentes (años 2012 y 2018). Los resultados muestran en general un incremento de la prevalencia de conductas sugestivas de TCA en el intervalo de tiempo considerado, desde un 6,43\% para el año 2012 hasta un 15,3\% en el año 2018. Un resultado muy superior a lo reportado recientemente por Kolar et al. (2016) en el que refieren prevalencia de alrededor del 1,6\% en jóvenes latinoamericanos y por Quintero et al (2003) en Venezuela, con tasa de $1,56 \%$ y más cercanos a los reportes de Ríos, Machado, Rísquez, \& Ortega (1994) de 18\%. No obstante, los hallazgos son consistentes con lo referido por Galmiche, Déchelotte, Lambert, \& Tavolacci (2019), en tanto confirman que los TCA, en general, son altamente prevalentes en todo el mundo y que estas tasas aumentaron durante el intervalo de estudio que ellos reportan: desde un 3,5\% para el período 2000-2006 hasta un 7,8\% para el período 2013-2018, patrón que se observa con la misma tendencia en los participantes de nuestro estudio y con un incremento porcentual para estudiantes masculinos en la muestra del año 2018, comparado con la muestra de 2012 (4,02\% vs $1,29 \%$ ), siendo significativas las diferencias para cada sexo en el contraste de los años 2012 vs 2018.

Los resultados de la investigación también revelan específicamente que las tasas de incremento de puntajes en el instrumento de medida empleado EAT-26, son estadísticamente significativas en el caso de las puntuaciones para el factor Bulimia y Control Oral, pero no para el caso de Anorexia. Sin embargo, para el punto de corte EAT-26 $\geq 20$ de conductas sugestivas de TCA, los datos muestran diferencias significativas tanto para el puntaje total del instrumento como para el factor Control Oral; cabe recordar que este factor del instrumento está asociado a la vigilancia de la ingesta de comida y observancia de presiones del entorno respecto a la conducta alimentaria. En el caso de los contrastes entre instituciones, se observaron diferencias estadísticamente significativas para los puntajes del EAT-26 en la escala total en cinco (5) institutos y específicamente diferencias para el puntaje total de la prueba en dos (2) institutos y en cuatro (4) para los factores Bulimia y Control Oral; en ningún instituto se observó diferencia significativa para el factor Dieta. No obstante, solo en dos (2) de los seis (6) institutos, considerando el punto de corte EAT-26 $\geq$ 20, se observaron diferencias significativas para el factor Dieta y para el factor Control Oral, siendo básicamente estables los puntajes entre instituciones en el tiempo bajo el referido criterio. 
Lo anterior coincide parcialmente con lo reportado por Kolar et al (2016) quienes refieren al respecto que en América Latina las tasas de prevalencia son comparables entre diferentes países. Según Kolar et al, la literatura reporta que, para el caso de la anorexia, la prevalencia ha tendido a mantenerse estable en los últimos años si se compara con muestras norteamericanas y europeas, lo que puede obedecer a diferencias en cuanto a ideales de la figura corporal que poseen las muestras de participantes latinos. En torno a ello, Warren, Gleaves y Rakhkovskaya (2013) refieren que la autoimagen personal en jóvenes estudiantes latinas sugiere actitudes más favorables a rasgos corporales más curvos y a un peso mayor que lo observado en otros grupos étnicos occidentales. La observación de diferencias en Control Oral y no en los otros factores medidos con el instrumento puede obedecer a un incremento de conductas impulsivas que van transformándose en comportamientos compulsivos y más habituales en los sujetos que incluso pueden llegar a ser deliberadamente planificados, tal como lo afirman Pearson, Wonderlich \& Smith (2015), y que serviría a los propósitos de reducir experiencias de afecto negativo en los adolescentes.

\section{Conclusiones}

Los análisis presentados permiten concluir en torno al objetivo propuesto. En efecto, se logró comparar a dos grupos de adolescentes, estudiantes de bachillerato a partir de datos del instrumento EAT26 tomados en dos años diferentes y en seis (6) instituciones educativas. En el caso de la muestra de nuestro estudio, se observaron promedios relativamente similares en el intervalo considerado, pero solo para el factor Dieta del EAT-26, sin incremento significativo en el periodo estudiado. Se encontraron puntajes más altos en 2018 para la muestra total y para el grupo de riesgo EAT-26 $\geq 20$ con puntajes sugestivos de TCA.

Los resultados apuntan hacia la importancia de considerar estos cambios reportados en torno a la prevalencia de conductas de riesgo de TCA en el estudio de los factores de riesgo que los incrementan en la población adolescente que habita contextos urbanos, a fin de diseñar efectivas políticas públicas de prevención, así como para la estimación de los costos asociados al diseño de programas de orientación y tratamiento de los factores relacionados al riesgo de TCA, en especial, al tratamiento de la bulimia y el trastorno por atracón y las conductas compulsivas de control. Los datos sugieren que, si bien las tasas reportadas para estos factores en el EAT-26 se mantienen relativamente estables, la prevalencia de conductas sugestivas de TCA en las muestras de jóvenes de interés resulta muy alta al contrastarla con el promedio latinoamericano; por tanto, indudablemente representan un riesgo de salud de considerable importancia que demanda y justifica la asignación de recursos para su atención. Nuevas investigaciones serán necesarias para continuar observando las modificaciones en los niveles de conductas de riesgo de TCA y en las tasas de prevalencia de los TCA en el grupo etario de interés.

\section{Bibliografía}

American Psychiatric Association (2013). Diagnostic and Statistical Manual of Mental Disorders $(D S M-V) .5^{\text {th }}$ Ed. Arlington: American Psychiatric Association.

Caldera, I. A., Martín, P., Caldera, J. F., Reynoso, O. U., \& Zamora, M. (2019). Predictores de conductas alimentarias de riesgo en estudiantes de bachillerato. Revista Mexicana de Trastornos Alimentarios, $\quad 10 \quad$ (1), 22-31. http://dx.doi.org/10.22201/fesi.20071523e.2019. 1.519

Cheah, S. L., Jackson, E., Touyz, S. \& Hay, P. (2020, en prensa). Prevalence of eating disorder is lower in migrants than in the Australian-born population. Eating Behaviors https://doi.org/10.1016/j.eatbeh.2020.101370

Galmiche, M., Déchelotte, P., Lambert, G., \& Tavolacci, M. P. (2019). Prevalence of eating disorders over the 2000-2018 period: a systematic literature review. The American Journal of Clinical Nutrition 109 (5), 14021413, https://doi.org/10.1093/ajcn/nqy342

Hernández, R., Fernández, C., \& Baptista, M. P. (2014). Metodología de la investigación (6ta Ed.). México: McGraw-Hill.

Hoek, H. W. (2014). Epidemiology of eating disorders in persons other than the highrisk group of young western females. Current Opinion in 
Psychiatry, 27 (6), 423-425. DOI: 10.1097/YCO.0000000000000104

Instituto Nacional de Estadística, INE (2014). XIV Censo nacional de población y vivienda: Resultados por Entidad Federal y Municipio del Estado Miranda. Caracas: Instituto Nacional de Estadística. Recuperado de http://www.ine.gov.ve/documentos/Demografia/ CensodePoblacionyVivienda/pdf/miranda.pdf

Keski-Rahkonen, A., \& Mustelin, L. (2016). Epidemiology of eating disorders in Europe: prevalence, incidence, comorbidity, course, consequences, and risk factors. Current Opinion in Psychiatry, 29 (6), 340-345. doi: 10.1097/YCO.0000000000000278

Khanh-Dao Le, L., Jan JBarendregt, J. J., Hay. P., \& Mihalopoulos, C. (2017). Prevention of eating disorders: A systematic review and metaanalysis. Clinical Psychology Review, 53, 46-58. doi: 10.1016/j.cpr.2017.02.001

Kolar, D. R., Rodriguez, D. L., Chams, M., \& Hoeck, H. W. Epidemiology of eating disorders in Latin America: a systematic review and meta-analysis. Current Opinion in Psychiatry, 29 (6), 363-371. https://doi.org/10.1097/YCO.000000000000027 9

Koushiou, M., Nikolaou, P., \& Karekla, M. (2019). Prevalence and Correlates of Eating Disorders in Greek-Cypriot Adolescents and Young Adults. The European Journal of Counseling Psychology, 8 (1). Recuperado de: https://ejcop.psychopen.eu/issue/view/13

Moreno, P. A., Ochoa, S. A., Vásquez, D., Ortiz, E., López, Y., Marin, L., \& García, A. (2016). Trastornos de la conducta alimentaria en adolescentes de colegios públicos de Pereira. Revista Médica de Risaralda, 22 (1), 9-13. Recuperado de https://revistas.utp.edu.co/index.php/revistamedi ca/article/view/9871

Pearson, C. M., Wonderlich, S. A., \& Smith, G. T. (2015). A risk and maintenance model for bulimia nervosa: from impulsive action to compulsive behavior. Psyuchological Review, 122 (2), 516-535. doi:10.1037/a0039268.

Ríos, A., Machado, T., Rísquez, F. y Ortega, M. (1994). Detención de actitudes Sugestivas de Anorexia y Bulimia Nerviosa en estudiantes de 10 a 18 años de edad, del Área Metropolitana de Caracas. Monografía no publicada. Conicit. Caracas.

Thomas, J. J., Lee, S., \& Becker, A. E. (2016). Updates in the epidemiology of eating disorders in Asia and the Pacific. Current Opinion in $\begin{array}{llll}\text { Psychiatry, } & 29 & \text { (6), 354-362. }\end{array}$ https://doi.org/10.1097/YCO.000000000000028 8

Urrutia, M. T., Cazenave, A., Badillo, H., \& Fajardo, O. (2008). Trastornos de la conducta alimentaria en adolescentes. Revista Horizonte de Enfermería, 19 (1), 13-21. Recuperado de http://publicaciones.horizonteenfermeria.uc.cl/in dex.php/rhe/article/view/669/503

Van Hoeken, D.' Burns, J. K.., \& Hoek, H. W. (2016) Epidemiology of eating disorders in Africa. Current Opinion in Psychiatry, 29 (6), 372-377. https://doi.org/10.1097/YCO.000000000000027 4

Vaidyanathan, S., Pooja, K., \& Menon, V. (2019). Eating Disorders: An Overview of Indian Research. Indian Journal of Psychological Medicine, $41 \quad$ (4), 311-317. doi: 10.4103/IJPSYM.IJPSYM_461_18

Quintero E., Pérez A. C., Montiel, C., Pirela, D., Acosta, M. F., \& Pineda, N. (2003). Prevalencia y características clínicas en adolescentes de la ciudad de Maracaibo, Estado de Zulia, Venezuela. Investigación Clínica, 44, 179-93.

Ríos, A., Machado, T., Rísquez, F. \& Ortega, M. (1994). Detención de actitudes Sugestivas de Anorexia y Bulimia Nerviosa en estudiantes de 10 a 18 años de edad, del Área Metropolitana de Caracas. Trabajo de investigación. Conicit. Caracas.

Walsh, B. T. (2019). Diagnostic Categories for Eating Disorders. Current Status and What Lies 
Trastornos del comportamiento alimentario; estudiantes de bachillerato; escala EAT-26; pruebas noparamétricas.

Ahead. Psychiatric Clinics of North America, 42 (1), $1-10$. https://doi.org/10.1016/j.psc.2018.10.001

Warren, C.S., Gleaves, D.H., \& Rakhkovskaya, L. M. (2013). Score reliability and factor similarity of the Sociocultural Attitudes Towards Appearance Questionnaire-3 (SATAQ-3) among four ethnic groups. Journal of Eating Disorders 1 (14). Recuperado de https://www.ncbi.nlm.nih.gov/pmc/articles/PMC 4081787/pdf/2050-2974-1-14.pdf 